\title{
Chronic Abdominal Pain in a Geriatric Patient: A Rare Case of a Spigellan Hernia
}

Thomas D. Wright, PhD', Olumayowa Dayo, MD², Lynne J. Goebel, MD'

ABSTRACT

We present a case of Spigelian hernia in a 77-year-old patient to highlight the difficulty in making this diagnosis and to raise awareness of this rare condition among physicians who care for geriatric patients with chronic bouts of abdominal pain. The patient presented to the emergency department with a two-day complaint of abdominal pain after three years of similar recurrent attacks.

Results of the physical examination and computed tomography (CT) of the abdomen were consistent with a left-sided Spigelian hernia. Operative repair was performed using robotic-assisted reduction and the patient recovered without any complications.
Author affiliations are listed at the end of this article.

Correspondence to: Lynne J. Goebel, MD Marshall Unviersity Joan C. Edwards School of Medicine goebel@marshall.edu

\section{KEYWORDS}

Robotic-assisted surgery, Chronic Abdominal Pain, Geriatric, Spigelian Hernia

\section{INTRODUCTION}

Spigelian hernia is a rare ventral abdominal defect along the semilunar line of the abdomen that can present with non-specific symptoms. It occurs as a wall defect between the lateral aspect of the rectus abdominis muscle and the medial aspect of the aponeurosis of the transversus abdominis muscle. ${ }^{1}$ It represents $1-2 \%$ of all hernias, is more common in women, and occurs more often in the 40-70 year age group. ${ }^{2,3,4}$ Spigelian hernias are congenital or acquired. Prior open abdominal surgery, especially for urologic or gynecologic reasons, has been linked to the development of a spigelian hernia. ${ }^{5}$

It is important to recognize cues in a patient's history and have a high level of suspicion to be able to diagnose and treat this hernia appropriately. Patients typically present with vague abdominal complaints and have a history of previous abdominal surgery. ${ }^{6}$ Since most patients present to their primary care physicians and emergency medicine physicians when a hernia becomes symptomatic, such physicians need to have spigelian hernia in their differential in order to avoid the morbidity from a missed diagnosis.

\section{CASE REPORT}

A 77-year-old woman presented to the emergency department with abdominal pain for two days. The patient reported feeling a hard, tender lump on the left side of her abdomen that was reducible with lying down. She had not had a bowel movement in two days. Her past medical history was significant for multiple abdominal surgeries including right ventral and umbilical hernia repairs with mesh. Her medical comorbidities included a history of breast cancer, hypertension, diabetes, anxiety disorder, and gastroesophageal reflux disease.

On chart review from 2013-2017, she presented to the walk-in clinic or emergency room four times with abdominal pain and self-reported suspicion of hernia problems. During most visits, she complained of left lower quadrant crampy pain with constipation that was followed by diarrhea. Previous work-up included serology for celiac disease, stool studies for Clostridioides difficile, enteric pathogens, ova and 


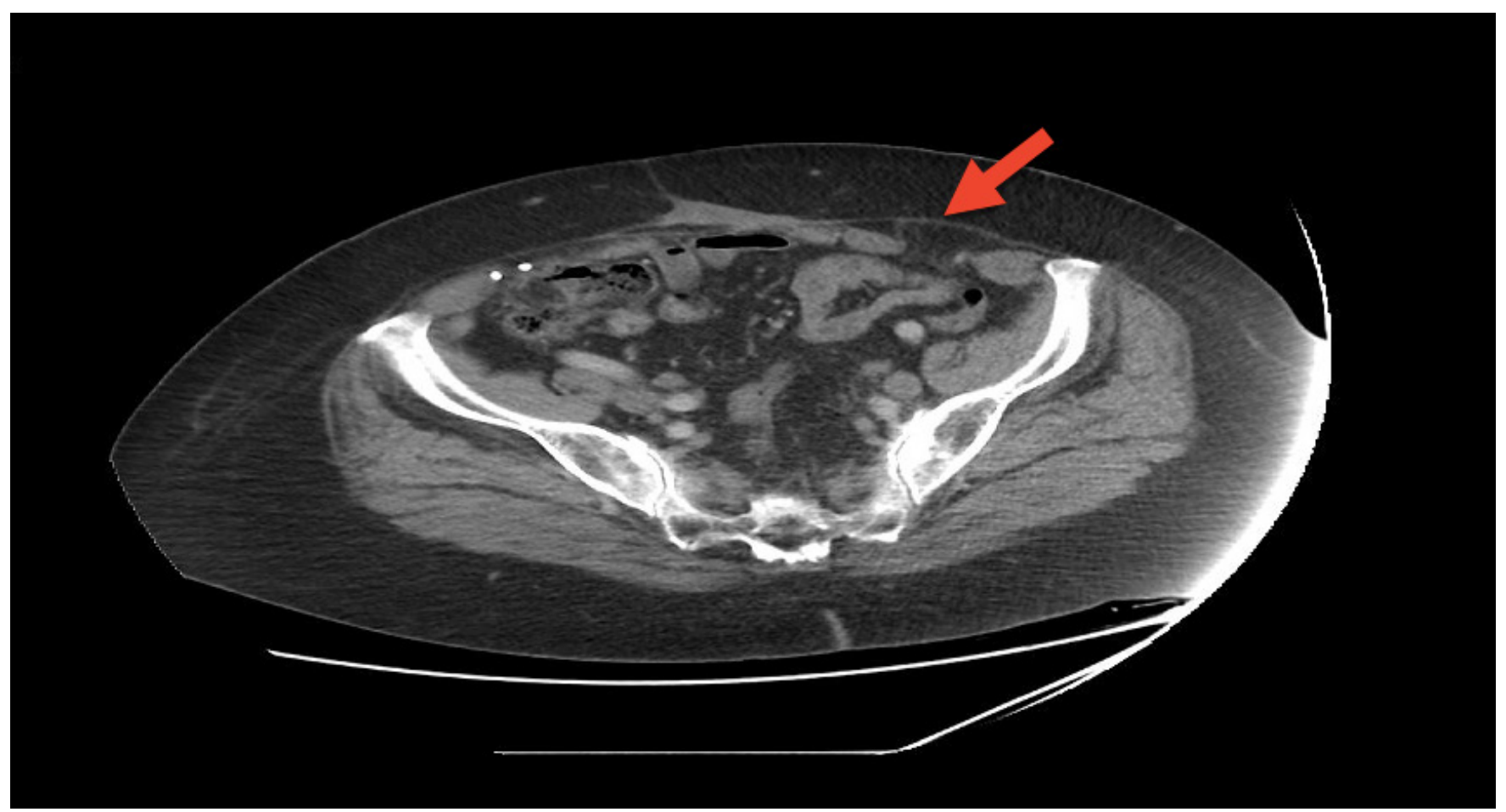

Figure 1. Axial abdominal CT image of a left spigelian hernia containing fat and a short segment of the left colon.

parasite, which were all negative. During a visit in 2015, she complained of left lower quadrant pain relieved by lying flat. Computed tomography (CT) imaging at the time revealed evidence of multiple previous abdominal surgeries; however, it failed to disclose a hernia.

During the current visit, physical exam revealed a soft, distended abdomen with a slightly tender palpable mass in the left lower abdomen. Based on the patient's clinical presentation and history of hernias, another abdominal CT was performed which revealed a new hernia along the left lower abdominal wall at the level of the left iliac crest, consistent with a spigelian hernia (Figure 1). The hernia contained fat and a short segment of the left colon. Therefore, a pre-operative diagnosis of colonic obstruction with spigelian hernia was made. The patient was taken to the operating room for hernia reduction.

A robotic-assisted lysis of adhesions and reduction of spigelian hernia with intraperitoneal overlay mesh was performed under general anesthesia. The surgical findings confirmed the preoperative diagnosis. The patient did well post-operatively and recovered without complications.

\section{DISCUSSION}

Our patient with spigelian hernia presented with vague recurrent abdominal symptoms, and initially had a normal physical exam making it hard to diagnose. A delay in diagnosis could lead to further morbidity since approximately $27 \%$ of spigelian hernias strangulate. ${ }^{2}$ This is a relatively high rate since the strangulation rate of all hernia types combined is 1-3\%.7 Although a physical exam is not very sensitive, a palpable mass along the semilunar line in a patient with risk factors may indicate spigelian hernia. This exam finding is seen in about half of cases as the hernia can be deep to the external oblique muscle making it hard to palpate on exam. Techniques that increase intra-abdominal pressure such as the Valsalva maneuver may be necessary to identify hernias that self-reduce with lying flat.

The following differential diagnoses should be considered when evaluating for spigelian hernia: lipoma and tumors of abdominal wall, incisional 
hernia, abdominal obstruction, ischemic bowel, diverticulitis, appendicitis, and hematoma of the rectus muscle. ${ }^{8}$ Differentiation between these diagnoses can be achieved primarily using CT or ultrasound imaging modalities. Specifically, standing ultrasound or CT imaging with oral contrast are preferred. ${ }^{7}$ Unfortunately CT is not always diagnostic, especially in the case of hernias that self-reduce. With high suspicion for the diagnosis, physicians could request for thin cuts through the spigelian hernia belt, improving the diagnostic sensitivity. Approximately $90 \%$ of Spigelian hernias occur in this region located six $\mathrm{cm}$ above the level of the anterior superior iliac spines. ${ }^{9}$

Risk factors for spigelian hernias include obesity, pregnancy, peritoneal dialysis, cirrhosis with ascites, COPD, collagen disorders such as Ehlers Danlos, constipation, rapid weight loss, previous abdominal surgery, and being elderly. ${ }^{7,9}$ Our elderly patient had many previous abdominal surgeries, constipation and obesity as her risk factors.

Methods of surgical repair include open and minimally invasive laparoscopic surgery. Some techniques include intraperitoneal onlay mesh, transabdominal preperitoneal repair, and total extraperitoneal repair. ${ }^{9}$ Total extraperitoneal mesh is the gold standard according to one source. ${ }^{6}$ However, since there are no randomized trials of repair techniques, there is no evidence-based best method at present. In this case, the hernia was successfully reduced with robotic-assisted surgery.

A review of 252 laparoscopically repaired cases of spigelian hernia showed a recurrence rate of $1 \%$ and a complication rate of $4.7 \% .{ }^{2}$ There are very few cases of robotic spigelian hernia repair with variable follow up. This technique was used in three other cases of Spigelian hernia, with no reports of intraoperative or postoperative complications. Patients reported minimal pain and hospitalization was no longer than overnight admission. ${ }^{10}$ Follow up, regarding the recurrence rate, for these three cases was not reported. Pirolla et al compared two cases of robotic repair to three cases of laparoscopic spigelian hernia repair. Robotic repair had the advantage of the 360-degree rotation of the robotic arm allowing ease of suturing the ring and stapling the mesh and reduction in tremor. ${ }^{8}$ Additionally, the robotic cases had less pain post-operatively and no recurrences at one year. There was an increased cost for robotic surgery of $\$ 3000$ per case but blood loss, surgical time and hospitalization were similar to laparoscopic repair. ${ }^{8}$

Three other recent case reports of spigelian hernia presented similarly to our patient, however, the contents of each hernia were different. ${ }^{11,12,13}$ In these cases, the surgical approaches were determined by the content and complexity of the hernia. Most commonly, contents of the hernia include omentum or intestine; others, however, contain ovary, fallopian tube, uterus, testis, gallbladder, stomach, Meckel's diverticulum, appendix, and bladder. ${ }^{8,10}$

\section{CONCLUSION}

A high level of suspicion is necessary to diagnose a spigelian hernia since patients present with vague symptoms and physical exam has low sensitivity for diagnosis. Physicians need to recognize the clinical setting in which spigelian hernias occur and consider it in their patients with a prior history of multiple abdominal surgeries or other risk factors to facilitate timely diagnosis and management.

\section{AUTHOR AFFILIATIONS}

1. Marshall University Joan C. Edwards School of Medicine, Huntington, West Virginia

2. Kaiser Permanente, Oakland, California

\section{REFERENCES}

1. Yau KK, Siu WT, Chan KL, Li KW. A man with recurrent lower abdominal pain: Spigelian hernia. Surg Laparosc Endosc Percutan Tech. 2008;Feb;18(1):106-8.

2. Rankin A, Kostusiak M, Sokker A. Spigelian Hernia: Case Series and Review of the Literature. Visc Med. 2019;35(2):133-136.

3. Fisher BL. Video-assisted spigelian hernia repair. Surg Laparosc Endosc. 1994;4:238-240.

4. Sachs M, Bojunga J. The so-called Spigelian hernia-a rare lateral hernia of the abdominal wall. Zentralbi Chir. 1998;123(3):267-271.

5. Khadka P, Dhakal SKS. Case report of ovary and 
fallopian tube as content of a Spigelian hernia - a rare entity. Int J Surg Case Rep. 2017;31:206-208.

6. Ussia A, Imperato F, Schindler L, Wattiez A, Koninckx PR. Spigelian hernia in gynaecology. Gynecol Surg. 2017;14(1):8.

7. Huttinger R, Sugumar K, Baltazar-Ford KS. Spigelian Hernia. [Updated 2019 Jun 10]. In: StatPearls [Internet]. Treasure Island (FL): StatPearls Publishing; 2020 Jan. Available from: https://www.ncbi.nlm.nih.gov/books/ NBK538290/

8. Pirolla,EH, Fregni F, Godoy-Santos AL, Schraibman V. A feasibility report of a novel and unpublished surgical approach for spiegel's hernia: reconstruction using robotics-assisted surgery. Advanced Health Care Technologies. 2015;:!3-12.

9. Mittal T, Kumar V, Khullar R, Sharma A, Soni V, Baijal M, Chowbey PK. Diagnosis and management of spigelian hernia: a review of literature and our experience. J Minim Access Surg. 2008;4(4):95-98.

10. Jamshidian M, Stanek S, Sferra J, Jamil T. Robotic repair of symptomatic spigelian hernias: a series of three cases and surgical technique review. J Robot Surg. 2018; 12(3):557-560.

11. Karkocha D, Lech G, Jankowski M, Slodkowski M. Left-sided spigelian hernia with nontypical hernia sac content. Pol Przegl Chir. 2019;91(6):4749.

12. Anilir E, Buyuker F, Tosun S, Alimoglu O. Incarcerated spigelian hernia: a rare cause of abdominal wall tender mass. North Clin Istanb. 2019;7(1):74-77.

13. Nicolas G, Emmanuel N, Charbel C, Awdeh A, Bitar B, Abourafeh SS, Farah M, Daher L, Mhanna $A A$, and Khoury G. An unusual case of left spigelian hernia containing part of fibroid uterus and the left adnexa in a 66-year-old woman. Am J Case Rep. 2019;20:1643-1647. 